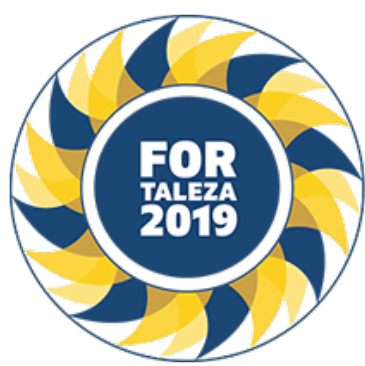

\title{
CORONARY ANEURYSMS: RESOLUTION AFTER TUBERCULOSIS TREATMENT
}

Nestor Barreto-Neto (Hospital das Clínicas da Faculdade de Medicina da Universidade de São Paulo, São Paulo, SP, Brasil), Alexandre Segre (Hospital das Clínicas da Faculdade de Medicina da Universidade de São Paulo, São Paulo, SP, Brasil), Aline Carolina Capellato Dias Baccaro (Hospital das Clínicas da Faculdade de Medicina da Universidade de São Paulo, São Paulo, SP, Brasil), Matheus Santos Rodrigues Silva (Hospital das Clínicas da Faculdade de Medicina da Universidade de São Paulo, São Paulo, SP, Brasil), Rodrigo de la Quintana Vidaurre (Hospital das Clínicas da Faculdade de Medicina da Universidade de São Paulo, São Paulo, SP, Brasil), Lissiane K Guedes (Hospital das Clínicas da Faculdade de Medicina

da Universidade de São Paulo, São Paulo, SP, Brasil), Luciana P C Seguro (Hospital das Clínicas da Faculdade de Medicina da Universidade de São Paulo, São Paulo, SP, Brasil), Eduardo F Borba (Hospital das Clínicas da Faculdade de Medicina da Universidade de São Paulo, São Paulo, SP, Brasil), Eloisa Silva Dutra de Oliveira Bonfa (Hospital das Clínicas da Faculdade de Medicina da Universidade de São Paulo,

São Paulo, SP, Brasil), Rosa Maria R Pereira (Hospital das Clínicas da Faculdade de Medicina da Universidade de São Paulo, São Paulo, SP, Brasil)

\section{BACKGROUND}

Coronary artery aneurysm (CAA) is a rare entity, with a prevalence of 0.1 to $4.9 \%$ in angiographic series. Atherosclerosis is the most common cause of CAA, but systemic vasculitides, particularly Kawasaki arteritis in children and polyarteritis nodosa and Takayasu arteritis in adults, are important differential diagnoses. Tuberculosis (TB) has been linked to vasculitis in some retrospective studies and is known to independently increase myocardial infarction risk through systemic inflammation.

\section{CASE REPORT}

A 60-year-old woman was admitted to a Rheumatology Inpatient Unit to investigate left anterior descending coronary artery (LAD) aneurysms, suggestive of coronaritis. One year before, she had experienced intense chest pain at rest and was diagnosed with a non-ST segment elevation myocardial infarction (NSTEMI). She was then submitted to catheterization of the coronary arteries, which demonstrated segmental aneurysms and $>90 \%$ stenosis of the middle portion of the LAD, as well as $>90 \%$ ostial stenosis of the second diagonal branch. At the time, she was treated with standard drug therapy for NSTEMI. She also had a history of erythema nodosum on both legs and was referred for investigation. Cultures of nodule exudates were positive for $M$. tuberculosis and treatment for TB was initiated four months after NSTEMI. Five months after initiation of TB treatment, CRP and ESR levels had significantly decreased (160 to $3.2 \mathrm{mg} / \mathrm{mL} ; 80$ to $22 \mathrm{~mm}$ 1st/h, respectively). ANA, ANCA, and other antibody tests, as well as viral serologies, were negative. Coronary CT-scan demonstrated a calcium score of zero, with no signs of atherosclerosis. New catheterization of coronary arteries, performed 12 months after NSTEMI, evidenced no signs of aneurysms, although critical stenosis of LAD remained. Furthermore, a $1.1 \mathrm{~cm}$ saccular aneurysm of the infra-renal aorta was identified. PET-CT did not detect active vascular inflammation.

The patient did not meet criteria for polyarteritis nodosa, giant-cell arteritis, or other systemic vasculitides, although none of them can be fully excluded. The patient is currently scheduled to perform coronary angioplasty to treat coronary stenosis followed by endovascular procedure to treat the aortic aneurysm.

\section{CONCLUSION}

Since the patient has no signs of atherosclerosis, it is highly suggestive that the evidenced coronaritis is associated with either inflammation or infection. Due to the resolution of aneurysm images and 
inflammatory parameters with only TB treatment, it is plausible to consider TB a possible cause or contributor to the formation of these aneurysms. Further studies are required to establish correlation between TB infection and medium and large-vessel aneurysms. 